\title{
AMCoR
}

Asahikawa Medical University Repository http://amcor.asahikawa-med.ac.jp/

Clin Chim Acta (2014.9) 437:129-135.

Non-transferrin-bound iron assay system utilizing a conventional automated analyzer.

Satoshi Ito, Katsuya Ikuta, Daisuke Kato, Kotoe Shibusa, NoriyasuNiizeki, Hiroki Tanaka, Lynda Addo, Yasumichi Toki, MayumiHatayama, Junki Inamura, Motohiro Shindo, Katsunori Sasaki, Naomilizuka, Mikihiro Fujiya, Yoshihiro Torimoto, Yutaka Kohgo 


\section{Non-transferrin-bound iron assay system utilizing a conventional automated analyzer}

Satoshi Ito ${ }^{\text {a }}$, Katsuya Ikuta ${ }^{a^{*} \rrbracket}$, Daisuke Kato ${ }^{\mathrm{b}}$, Kotoe Shibusa ${ }^{\mathrm{a}}$, Noriyasu Niizeki $^{\mathrm{c}}$, Hiroki Tanaka ${ }^{\mathrm{d}}$, Lynda Addo ${ }^{\mathrm{a}}$, Yasumichi Toki ${ }^{\mathrm{a}}$, Mayumi

Hatayama $^{a}$, Junki Inamura ${ }^{a}$, Motohiro Shindo ${ }^{\text {a }}$, Katsunori Sasaki ${ }^{d}$, Naomi Iizuka $^{\mathrm{b}}$, Mikihiro Fujiya ${ }^{\mathrm{a}}$, Yoshihiro Torimoto ${ }^{\mathrm{e}}$, Yutaka Kohgo ${ }^{\mathrm{a}}$

${ }^{a}$ Division of Gastroenterology and Hematology/Oncology, Department of Medicine, Asahikawa Medical University, Asahikawa, Hokkaido, Japan

${ }^{\mathrm{b}}$ Research and Development Department, Shino-Test Corporation, Sagamihara, Kanagawa, Japan

${ }^{\mathrm{c}}$ Department of Medical Laboratory and Blood Center, Asahikawa Medical University Hospital, Asahikawa, Hokkaido, Japan

${ }^{\mathrm{d}}$ Department of Gastrointestinal Immunology and Regenerative Medicine, Asahikawa Medical University, Asahikawa, Hokkaido, Japan

${ }^{\mathrm{e}}$ Oncology Center, Asahikawa Medical University Hospital, Asahikawa, Hokkaido, Japan

Contact information for correspondence:

Katsuya Ikuta, M.D., Ph.D.

Assistant Professor

Division of Gastroenterology and Hematology/Oncology

Department of Medicine, Asahikawa Medical University

2-1-1-1 Midorigaoka-Higashi, Asahikawa, Hokkaido 078-8510, Japan

Tel: +81-166-68-2462 
Fax: $\quad+81-166-68-2469$

E-mail:ikuta@asahikawa-med.ac.jp

" SI and KI contributed equally to this manuscript. 


\begin{abstract}
Iron is an essential metal in the body, but its excessive accumulation causes damage in various organs through free radical production. Iron homeostasis is therefore tightly regulated. However, when iron balance collapses, such as in prolonged transfusion, transferrin (Tf) is fully saturated and non-Tf-bound iron (NTBI) appears in the serum. Monitoring serum NTBI levels is therefore crucial in assessment of the clinical status of patients with iron overload, since NTBI is associated with cellular and organ damage. Several methods for NTBI determination have been reported, but these are extremely complicated and very few laboratories can quantify NTBI at present. In this study, we established a novel assay system utilizing automated analyzers that are widely used in clinical laboratories for diagnostic testing. In this assay, NTBI is chelated by nitrilotriacetic acid (NTA), after which the iron is reduced and transferred to nitroso-PSAP, a chromogen. The assay shows excellent linearity, reproducibility, and compatibility with HPLC, one of the most reliable conventional methods for NTBI quantification. Our novel method for NTBI measurement is high-throughput and may be a useful and powerful tool in study of the physiological and clinical importance of NTBI.
\end{abstract}

Key words: Iron overload, iron toxicity, non-transferrin-bound iron, quantification, automated analyzer, nitroso-PSAP 


\section{List of abbreviations}

Tf: transferrin

NTBI: non-transferrin-bound iron

TfR1: transferrin receptor 1

HPLC: high-performance liquid chromatography

NTA: nitrilotriacetic acid

Nitroso-PSAP: 2-nitroso-5-[ $N$-n-propyl- $N$-(3-sulfopropyl)-amino] phenol

Bathophenanthroline: 4,7-diphenyl-1,10-phenanthroline

Ferene: 3-(2-pyridyl)-5,6-di(2-furyl)-1,2,4-triazine-5',5"'-disulfonic acid disodium salt

Ferrozine: 3-(2-pyridyl)-5,6-bis(4-sulfophenyl)-1,2,4-triazine

$\mathrm{R} 1$ : reagent 1

$\mathrm{R} 2$ : reagent 2

BSA: bovine serum albumin

sFe: serum iron

LOB: limit of blank

LOD: limit of detection

LOQ: limit of quantification

UIBC: unsaturated iron binding capacity

CKD: chronic kidney disease 


\section{Introduction}

Iron is an essential metal in the body, but iron overload causes organ damage through free radical production. Iron homeostasis is therefore tightly regulated under normal conditions by orchestration of proteins related to iron metabolism [1-4]. Iron circulates in serum by binding to its specific carrier protein, transferrin (Tf). In healthy individuals, only $20-30 \%$ of Tf is usually saturated with iron, so that during a period of iron burden (such as in red blood cell transfusion or iron administration) iron is immediately trapped by iron-free apo-Tf. However, in situations where the balance of iron metabolism collapses, Tf is fully saturated and non-Tf-bound iron (NTBI) appears in serum.

NTBI refers to all forms of iron in plasma that is not bound to Tf and was first measured by Hershko et al. in 1978 [5]. Iron binds to various ligands other than Tf, including albumin and citrate, implying that NTBI is present in heterogeneous forms [6-8]. However, these binding affinities are extremely weak compared to the iron-Tf complex and so NTBI can also be considered as "free iron" in serum. Tf-bound iron is taken up via a specific receptor, transferrin receptor 1 (TfR1), which is expressed in almost all cells and permits strict regulation of the cellular delivery of Tf-bound iron. In contrast, there is no known regulatory mechanism for NTBI uptake, and thus NTBI can easily enter cells and produce free radicals through the Fenton reaction, resulting in cell and organ damage $[9,10]$. The fraction of NTBI that is both redox-active and susceptible to chelation is sometimes referred to as labile plasma iron (LPI). LPI is considered to be a major component of NTBI, and is responsible for cytotoxic activity [11]. 
Clinically, NTBI is increased during iron overload in various disease states [12]. However, NTBI is decreased below the detection limit by long-term therapy with iron chelators such as deferoxamine and deferasirox in thalassemic patients and by venesection in hemochromatotic patients $[13,14]$. Therefore, measurement of NTBI levels is important for evaluating and monitoring the risk of iron toxicity [15]. Several methods for NTBI determination have been established [16-20], but it is still difficult to measure NTBI in blood due to the complex nature of the procedures involved and difficulty in eliminating iron contamination in reagents and equipment. Because of these problems, only a limited number of institutions can quantify NTBI at present.

We first focused on an NTBI assay using high-performance liquid chromatography (HPLC). The principle of this method is based on Gosriwatana et al. [21]: NTBI in serum is initially chelated by nitrilotriacetic acid (NTA) and then transferred to another chelator, CP22, which produces a chromogenic effect when bound to iron. The level of NTBI is quantified by measuring the peak absorbance at $450 \mathrm{~nm}$ using a non-metal HPLC system. However, the sensitivity of previously reported HPLC-based methods are considered to be unsatisfactory, as these methods cannot be used to measure serum samples containing low concentrations of NTBI, especially samples from healthy individuals. We previously reported that iron contamination during HPLC-based assay procedures can cause elevated blank values, which leads to a decrease in its analytical sensitivity. By extensive reduction of contaminated iron from the reagents and HPLC devices, we achieved very low reagent blanks and successfully improved the sensitivity of our HPLC method, as a result of which we were able to detect serum NTBI in normal individuals [22]. 
However, our improved non-metal HPLC method for NTBI quantification still needs further modification and may not be suitable for routine laboratory testing because of the following reasons: (i) expensive materials such as CP22, columns and non-metal HPLC devices are required for analysis, (ii) a multi-step sample pretreatment is required, and the whole procedure takes 2 hours to complete, (iii) the assay rate is therefore quite limited. These problems prevent handling of a large number of clinical samples and make it difficult to apply NTBI analysis in clinical practice.

To make NTBI quantification more convenient, an automated system that can be used in clinical laboratories for diagnostic testing is desired. Some advantages of the automated NTBI quantification method are :(i) simple sample preparation without complicated sample pretreatment steps, (ii) easy usability without special techniques and devices, (iii) applicability for widely-used automated biochemical analyzers, and (iv) a high-throughput assay at a low cost.

In the present study, we have developed a novel automated system for NTBI quantification and carried out analytical validations. 


\section{Materials and Methods}

\subsection{Chromogen screening}

The chromogens used were 2-nitroso-5-[N-n-propyl- $N$-(3-sulfopropyl)-amino] phenol (nitroso-PSAP) (Dojindo); 4,7-diphenyl-1,10-phenanthroline (Bathophenanthroline) (Dojindo); 3-(2-pyridyl)-5,6-di(2-furyl)-1,2,4-triazine-5',5"disulfonic acid disodium salt (Ferene) (Sigma); and 3-(2-pyridyl)-5,6-bis(4sulfophenyl)-1,2,4-triazine (Ferrozine) (Dojindo).

Standard metal solutions (iron, copper, cobalt and nickel) (Wako) were adjusted to $2 \mathrm{mM}$, and mixed with chromogens to make a final metal concentration of $20 \mu \mathrm{M}$. The spectrum of these chromogen-metal mixtures was scanned.

\subsection{Reagents for automated analysis}

Reagent-1 (R1) was prepared by mixing Tris buffer and NTA (iron-mobilizing ligand). Reagent-2 (R2) contained Tris buffer, ascorbic acid and nitroso-PSAP (ironchelating chromogen). R1 and R2 were titrated to $\mathrm{pH} 8.0$ and loaded onto automated analyzers. Final concentration of Tris buffer, NTA, ascorbic acid and nitroso-PSAP were set at $0.1 \mathrm{M}, 0.07 \mathrm{mM}, 1.32 \mathrm{mM}$ and $0.09 \mathrm{mM}$, respectively. Reagents were kept stable for at least 6 months by storing at $10^{\circ} \mathrm{C}$.

2.3. Settings for the automated analyzer and assay method NTBI was measured using the HITACHI 7700 Clinical Analyzer with the following settings: sampling volume: Sample / R1 / R2 $=15 \mu \mathrm{L} / 150 \mu \mathrm{L} / 50 \mu \mathrm{L}$; assay wavelength: Main / Sub $=750 \mathrm{~nm} / 600 \mathrm{~nm}$; assay method: 2-point end; reading point: first / last $=16$ / 34. In the assay method, samples and R1 (NTA) were first 
dispensed into reaction cells on the automated analyzer. After mixing and 5-minute incubation, R2 (nitroso-PSAP with ascorbic acid) was added to the cells and left for 5 more minutes. The increase in absorbance at $750 \mathrm{~nm}$ was used to calculate the amount of NTBI present in serum samples. Total assay time was only 10 minutes for each sample. 2-point linear regression calibration function was used for the analysis. The calibration stability was confirmed to be stable at least for 6 months. In the experiments using blood samples from human, serum was selected as an acceptable sample type; plasma samples obtained with heparin showed substantially equal values compared with serum (data not shown), but plasma samples obtained with citrate or ethylenediaminetetraacetic acid were considered to be inappropriate because these anticoagulants chelate iron.

2.4. Measurement of NTBI and Tf-bound iron by the automated system

The iron contents of iron citrate (Shino-test), 5\% bovine serum albumin (BSA) (Nacalai Tesque) and human holo-Tf (Sigma) were determined using a serum Fe (sFe) assay kit, which utilizes nitroso-PSAP as a chromogen (QuickAuto Neo Fe, Shinotest). NTBI concentration in these samples was also measured by the automated system and the percentage of NTBI to the iron contents was calculated as the recovery rate of NTBI.

\subsection{Dialysis of holo-Tf solution}

Human holo-Tf solutions ( 15 or $70 \mu \mathrm{M}$ iron was bound) were dialyzed with 50 $\mathrm{mM}$ Tris buffer ( $\mathrm{pH}$ 8.0) using dialysis tubing (EIDIA Co., Ltd) for three times to remove contaminated iron. Iron and NTBI levels in the dialyzed holo-Tf were 
quantified by sFe kit and the automated system, respectively. The percentage of NTBI to the iron contents was calculated as the recovery rate of NTBI.

\subsection{Intra- and inter-assay CVs}

The intra-assay CV was established using serum samples with low $(0.35 \mu \mathrm{M})$, medium $(0.55 \mu \mathrm{M})$ and high $(0.95 \mu \mathrm{M})$ NTBI levels. Each sample was measured in 20 replicates in the same run. The inter-assay CV was established using the same serum samples mentioned above. Samples were measured in 5 replicates for 20 days.

2.7. Limit of blank (LOB), limit of detection (LOD) and limit of quantification (LOQ)

LOB and LOD were defined as LOB $=$ Meanblank $+1.645 \times($ S.D.blank $)$ and

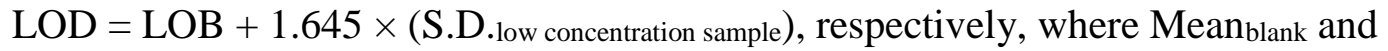
S.D.blank are the mean and S.D. of 6 blank samples assayed in 5 replicates for 5 days, and S.D.low concentration sample is the S.D. of 10 low concentration samples assayed in 5 replicates for 5 days. LOQ was determined as the lowest concentration that results in a CV of 20\%. Samples for LOQ determination were assayed in 5 replicates for 5 days.

\subsection{Linearity of the assay}

Linearity was assessed by serial dilution of $20 \mu \mathrm{M}$ iron citrate. Each dilution point was assayed in 5 replicates and S.D. was calculated.

\subsection{Tf saturation and NTBI level}

Pooled human serum was mixed with iron citrate $(0-75 \mu \mathrm{M})$ to examine the relationship between $\mathrm{Tf}$ saturation and NTBI. Tf saturation and NTBI were also 
assessed in serum samples obtained from 25 patients in Asahikawa Medical University Hospital, Hokkaido, Japan. All subjects signed an informed consent form in accordance with the Declaration of Helsinki. sFe and unsaturated iron binding capacity (UIBC) were quantified using commercial kits (QuickAuto Neo Fe and QuickAuto Neo UIBC, Shino-test). Tf saturation (\%) was calculated as $\mathrm{sFe} /(\mathrm{sFe}+$ $\mathrm{UIBC}) \times 100$.

2.10. Interference of hemolysis with the assay system

Red blood cell fraction was collected from healthy individual and treated with distilled water to lyse the blood cells. By adding saline to the hemolyzed sample, hemoglobin concentration was adjusted to 5,000 mg/dL. Then, pooled human serum was spiked with the hemoglobin solution or saline to make the final hemoglobin concentration of $0,100,200,300,400$ and $500 \mathrm{mg} / \mathrm{dL}$. The NTBI level was determined for each sample.

2.11. Correlation between results from the automated system and the HPLC method NTBI in serum samples from 33 patients and NTA-Fe $(0-17 \mu \mathrm{M})$ were measured by the automated system and our previously reported HPLC method [22]. Results obtained from these two methods were compared. 


\section{Results}

3.1. Absorbance spectrum of iron-chelating chromogens conjugated with metal ions $\mathrm{Fe}^{2+}$ chelated by nitroso-PSAP showed a single absorbance peak at $750 \mathrm{~nm}$. $\mathrm{Cu}^{2+}, \mathrm{Co}^{2+}$ and $\mathrm{Ni}^{2+}$ also bound to nitroso-PSAP, but had maximum absorption wavelengths at about $500 \mathrm{~nm}$ that were clearly distinguishable from the $\mathrm{Fe}^{2+}$ peak (Fig. 1A). Complexes of $\mathrm{Fe}^{2+}$ with Bathophenanthroline, Ferene and Ferrozine had maximum absorption peaks around $550-600 \mathrm{~nm} . \mathrm{Cu}^{2+}$ showed a broad absorption curve when bound to these three chromogens and overlapped with $\mathrm{Fe}^{2+}$ at $550-600 \mathrm{~nm}$, which could interfere with iron quantification and may lead to overestimation of NTBI (Fig. 1B, C, D). Thus, among the four chromogens, only the nitroso-PSAP complex at $750 \mathrm{~nm}$ could differentiate $\mathrm{Fe}^{2+}$ from $\mathrm{Cu}^{2+}$. Furthermore, the molar extinction coefficient of nitroso-PSAP was approximately twice that of the other chromogens. Because of these advantages, nitroso-PSAP was chosen for quantification of NTBI.

\subsection{Development of NTBI assay reagents}

NTBI assay reagents suitable for use in an automated analyzer were developed (Fig. 2). R1 containing NTA was added to serum samples directly to allow NTA chelation of iron from NTBI. The $\mathrm{pH}$ of the solution was changed to slightly alkaline ( $\mathrm{pH} 8.0$ ), in which NTA cannot chelate iron from diferric Tf. After addition of R1, the mixture was incubated for 5 min at $37^{\circ} \mathrm{C}$, after which $\mathrm{R} 2$ was added. In the NTA-Fe complex, iron is in the ferric form $\left(\mathrm{Fe}^{3+}\right)$, and thus is reduced to the ferrous form $\left(\mathrm{Fe}^{2+}\right)$ by the reducing agents in R2. After incubation for $5 \mathrm{~min}$, nitroso-PSAP binds to 
$\mathrm{Fe}^{2+}$ and the NTBI level can then be determined by measuring the absorbance of the nitroso-PSAP-Fe ${ }^{2+}$ complex at $750 \mathrm{~nm}$.

3.3. The automated system detects NTBI but not Tf-bound iron

To test whether our automated system can differentiate NTBI from Tf-bound iron, the NTBI recovery rate was investigated using iron citrate and 5\% BSA as a model for NTBI and human holo-Tf solution as Tf-bound iron. sFe assay kit showed that the iron contents in iron citrate, 5\% BSA and human holo-Tf solutions were 1.02 $\mu \mathrm{M}, 1.04 \mu \mathrm{M}$ and $15 \mu \mathrm{M}$, respectively. Then, NTBI concentration in these samples was determined by the automated system. The average NTBI recovery rates of iron citrate and 5\% BSA were $95.5 \%$ and $96.3 \%$, respectively. In contrast, only $2.1 \%$ of NTBI $(0.32 \mu \mathrm{M})$ was detected from a diferric Tf solution (Fig. 3).

\subsection{NTBI after dialysis of holo-Tf solution}

After thorough dialysis, NTBI level in the holo-Tf (15 $\mu \mathrm{M}$ iron-bound) was decreased to $0.77 \%$ or $0.14 \mu \mathrm{M}$. To simulate fully saturated transferrin in human serum, we additionally prepared $70 \mu \mathrm{M}$ iron-bound holo-Tf and it was dialyzed as the same procedure. NTBI level in the dialyzed holo-Tf was found to be $0.34 \%$ or 0.24 $\mu \mathrm{M}$.

3.5. Intra- and inter-assay CVs

The intra-assay CVs of samples with low $(0.35 \mu \mathrm{M})$, medium $(0.55 \mu \mathrm{M})$ and high $(0.95 \mu \mathrm{M})$ NTBI levels were $9.5 \%, 8.2 \%$ and $6.2 \%$, respectively. The inter-assay 
CVs during measurements over a 20-day period were $8.7 \%(0.35 \mu \mathrm{M}), 5.8 \%(0.55$ $\mu \mathrm{M})$ and $4.6 \%(0.95 \mu \mathrm{M})$.

3.6. LOD, LOQ and linearity of the automated system

The LOD and LOQ of the automated assay system were $0.34 \mu \mathrm{M}$ and $0.48 \mu \mathrm{M}$, respectively. Linearity was assessed up to $20 \mu \mathrm{M}$ NTBI. NTBI levels measured with the automated system plotted against concentrations of iron citrate gave a slope of 1.010, intercept of -0.017 , and $r^{2}$ of 0.999 (Fig. 4).

\subsection{Relationship between transferrin saturation and NTBI}

A known concentration of $\mathrm{Fe}^{3+}$ was added to pooled human serum to assess the relationship between transferrin saturation and NTBI. The rise in transferrin saturation was proportional to the amount of spiked iron, whereas NTBI only increased markedly as transferrin saturation reached $100 \%$ (Fig. 5A). In measurement of transferrin saturation and NTBI levels in 25 patients, those with transferrin saturation $>80 \%$ had higher NTBI, consistent with the data in the spiked samples (Figure. 5B).

\subsection{Interference of hemolysis with the assay system}

Iron mobilization from hemoglobin was investigated by measuring the NTBI value of hemoglobin-spiked pooled serum. NTBI levels in hemolytic samples (0-500 $\mathrm{mg} / \mathrm{dL}$ hemoglobin) were not substantially changed (Fig.6).

3.9. Correlation between results from the automated system and the HPLC method 
The automated system was compared with the HPLC method [22] by measuring NTBI in the same serum samples and NTA-Fe using the two methods. NTBI concentrations obtained with the automated system were plotted against those obtained with the HPLC method. For serum samples, the plot had a slope of 0.907 , intercept of 0.178 , and $\mathrm{r}^{2}$ of 0.538 (Fig. 7A). For NTA-Fe, slope, intercept and $\mathrm{r}^{2}$ were 1.058, 0.258 and 0.990 , respectively (Fig. 7B). When the data obtained from serum and NTA-Fe were combined, slope, intercept and $\mathrm{r}^{2}$ were 1.069, 0.083 and 0.983, respectively (Fig. 7C). 


\section{Discussion}

For the past few decades, serum NTBI had been quantified by several methods. A bleomycin assay, fluorescent probes, colorimetry and HPLC have all been used to monitor serum NTBI, but the reference values in healthy individuals obtained from these methods tend to be negative or described as 'not detected' [23-26]. This is mainly due to the high blank value, which is caused by iron contamination in assay reagents or analytical devices. In a previous report, we focused on the wellcharacterized HPLC method and improved the sensitivity by reducing background iron contamination [22]. We successfully measured serum NTBI without encountering negative values in healthy individuals $(0.206 \pm 0.091 \mu \mathrm{M}$ for males and $0.212 \pm 0.095 \mu \mathrm{M}$ for females). However, the HPLC method requires expensive materials and complicated sample pretreatment steps. These obstacles prevent simple and rapid measurement of serum NTBI in clinical laboratories. Thus, to permit NTBI quantification in daily laboratory testing, we developed NTBI assay reagents for use in automated analysis.

We chose nitroso-PSAP as a chromogen because of its high molar extinction coefficient and iron-specific maximum absorption of $750 \mathrm{~nm}$ (Fig. 1). Reagents, R1 and R2 contain NTA and nitroso-PSAP, respectively (Fig. 2). NTBI is present in heterogeneous forms, with the main fractions being albumin-bound iron and citratebound iron [6-8]. Our automated system can quantify these forms of iron as NTBI with the recovery rate of $>95 \%$. On the other hand, the detectable amount of NTBI from holo-Tf did not reach to the LOD, which can be considered as 'not detected'. These data suggest that the automated system can clearly distinguish NTBI from Tf- 
bound iron and possible false positive caused by iron mobilization form holo-Tf can be ignored (Fig. 3).

In patients with iron overload, the serum NTBI level can exceed $4 \mu \mathrm{M}$ [23-26]. Therefore, we investigated the assay range of the automated system. The results in Fig. 4 show that the linearity was reliable up to $20 \mu \mathrm{M}$, which should be a sufficient dynamic range to analyze patient serum with higher NTBI values. We also confirmed that inter- and intra-assay precision was satisfactory, indicating that the NTBI values obtained with our automated system are highly reproducible. Using the methods described by Armbruster et al. [27], LOD and LOQ were defined as $0.34 \mu \mathrm{M}$ and 0.48 $\mu \mathrm{M}$, respectively. Since NTBI levels in healthy individuals range from -0.30 to 0.76 $\mu \mathrm{M}$, with some differences among quantification systems [28-32], the detectable serum NTBI may be lower than the defined LOD. However, in patients with iron overload, there is a significant rise in serum NTBI to much higher levels than those in healthy people: $0.761 \pm 0.504 \mu \mathrm{M}$ in hereditary hemochromatosis [30], $3.90 \pm 2.48$ $\mu \mathrm{M}$ in beta-thalassemia [25] and $1.9 \pm 2.1 \mu \mathrm{M}$ in sickle cell disease [26]. Thus, our automated system can detect elevated serum NTBI and should serve as a useful tool for monitoring iron toxicity, especially in iron-overloaded patients.

When pooled human serum was loaded with iron, a significant amount of NTBI was detected after Tf was fully saturated (Fig. 5A). In a biological serum matrix, our automated system successfully quantified iron that is not bound to Tf. The results obtained from 25 human serum samples also showed that NTBI levels increased rapidly after Tf saturation reached $80 \%$, which was consistent with the data obtained above. However, several serum samples had relatively high NTBI levels of $>0.5 \mu \mathrm{M}$, even though Tf saturation was within a normal range (Fig. 5B). Similar observations 
have previously been reported [16, 21, 31, 33, 34, 35], but the mechanism of this phenomenon is currently uncertain.

We speculate that hemolysis might be involved in these unexpected results. In hemolytic samples, iron bound to hemoglobin could be chelated with NTA and transferred to nitroso-PSAP, which consequently leads to 'false positive' NTBI values. To investigate possible interference of hemolysis with the assay system, we prepared human pooled serum with different degrees of hemolysis and determined NTBI levels for each sample. The results showed that NTBI levels in these samples were not changed consistently (Fig. 6). This suggests that hemolysis itself does not increase the amount of NTBI in our automated system and is not the main reason for the elevated NTBI level with normal Tf saturation.

It is also possible that the serum concentration of albumin, citrate, phosphate or other molecules that bind free iron may affect the form of NTBI. Recently, it has been shown that in patients with chronic kidney disease (CKD), NTBI can occur at a low sFe level and normal Tf saturation. CKD patients show hyperphosphatemia due to reduced excretion of phosphate. Elevated serum phosphate might form a $\mathrm{Fe}^{3+}$ phosphate complex, which is one type of NTBI, and disrupt $\mathrm{Fe}^{3+}$ loading onto apo-Tf [36]. This model could explain the higher serum NTBI levels under normal Tf saturation, but further investigation in a larger number of samples will be needed to address these findings.

The correlation coefficient for NTBI measurement using the automated system and non-metal HPLC [22] was satisfactory and the automated system was shown to be compatible with the HPLC method (Fig. 7A, B, C). In the conventional HPLC method, apo-Tf needs to be fully saturated by cobalt ions before NTA is mixed with 
serum samples to avoid transfer of iron from the NTA-Fe complex to apo-Tf $[9,10]$. Without pre-saturation of apo-Tf with cobalt, iron mobilization from the NTA-Fe complex to apo-Tf would occur, resulting in the underestimation of NTBI values [21].

Since our automated system does not include this apo-Tf pretreatment, it is possible that the NTA-Fe complex donates iron to apo-Tf, which could lead to underestimation of NTBI. To investigate if iron was transferred from the NTA-Fe complex to apo-Tf, human serum samples (Tf saturation of $21 \%$ to $95 \%$ ) were prepared. NTBI in these samples was measured using the automated system with different sample-NTA incubation periods (1, 4 and $10 \mathrm{~min})$. Iron is loaded onto apoTf in a time-dependent manner, so if the iron transfer would take place in the sampleNTA mixture, NTBI level should be decreased as the reaction time extends. However, the results showed that NTBI levels did not change if sample-NTA reaction time were prolonged to $10 \mathrm{~min}$ (data not shown). These data indicate that in the automated system, iron bound to NTA was not substantially transferred to vacant iron-binding sites of apo-Tf, presumably because of the short (5 min) NTA reaction time (Fig. 2).

We previously investigated NTBI in patients after adult allogeneic hematopoietic cell transplantation using a non-metal HPLC method [37]. The results showed that serum ferritin seemed to be a better surrogate marker of iron overload than NTBI. However, we could not fully quantify a large number of serum samples because of the low assay rate of the non-metal HPLC method used in the previous study. Depending on their pathophysiological backgrounds, patients after hematopoietic transplantation sometimes have various attendant problems, such as infections, graft-versus-host disease, immunosuppressive drug administration, malnutrition, liver dysfunction, renal injury, and repeated transfusions. Further 
follow-up and more detailed analysis with a larger number of clinical samples will therefore be necessary to elucidate the clinical significance of NTBI in these conditions. From this point of view, our novel automated NTBI quantification system should produce more precise information.

The main objective of this study was to establish NTBI assay reagents that can be used in automated analyzers. The automated system does not require sample pretreatment steps, allowing completely automated and rapid analysis of serum NTBI. Analytical validation of the reagents demonstrated that NTBI quantification can be carried out reproducibly in a total reaction time of 10 minutes, with an assay rate of several hundreds of tests per hour using widely-used automated biochemical analyzers. Data reliability was also well-supported by the study using non-metal HPLC as a reference method.

Based on these results, we conclude that our automated system can be used practically for routine analysis and may be a powerful tool for studying the physiological and clinical importance of NTBI. 


\section{List of all authors:}

Satoshi Ito, M.D., Ph.D.

Division of Gastroenterology and Hematology/Oncology, Department of Medicine, Asahikawa Medical University, 2-1-1-1 Midorigaoka-Higashi, Asahikawa, Hokkaido 078-8510, Japan; e-mail: s-ito@asahikawa-med.ac.jp Katsuya Ikuta, M.D., Ph.D.

Division of Gastroenterology and Hematology/Oncology, Department of Medicine, Asahikawa Medical University, 2-1-1-1 Midorigaoka-Higashi, Asahikawa, Hokkaido 078-8510, Japan; e-mail: ikuta@asahikawa-med.ac.jp Daisuke Kato

Research and Development Department, Shino-Test Corporation, 2-29-14, Oonodai, Minamiku, Sagamihara, Kanagawa 229-0011, Japan; e-mail: daisuke.kato@shino-test.co.jp

Kotoe Shibusa

Division of Gastroenterology and Hematology/Oncology, Department of Medicine, Asahikawa Medical University, 2-1-1-1 Midorigaoka-Higashi, Asahikawa, Hokkaido 078-8510, Japan; e-mail: shibusa@asahikawamed.ac.jp

Noriyasu Niizeki

Department of Medical Laboratory and Blood Center, 2-1-1-1 MidorigaokaHigashi, Asahikawa, Hokkaido 078-8510, Japan; e-mail: niizeki@asahikawamed.ac.jp

Hiroki Tanaka, Ph.D.

Department of Gastrointestinal Immunology and Regenerative Medicine, Asahikawa Medical University, 2-1-1-1 Midorigaoka-Higashi, Asahikawa, Hokkaido 078-8510, Japan; e-mail: hiroki-t@ asahikawa-med.ac.jp Lynda Addo

Division of Gastroenterology and Hematology/Oncology, Department of Medicine, Asahikawa Medical University, 2-1-1-1 Midorigaoka-Higashi, Asahikawa, Hokkaido 078-8510, Japan; e-mail: lyndaddo@asahikawamed.ac.jp 
Yasumichi Toki, M.D.

Division of Gastroenterology and Hematology/Oncology, Department of Medicine, Asahikawa Medical University, 2-1-1-1 Midorigaoka-Higashi, Asahikawa, Hokkaido 078-8510, Japan; e-mail: toki@asahikawa-med.ac.jp Mayumi Hatayama, M.D.

Division of Gastroenterology and Hematology/Oncology, Department of Medicine, Asahikawa Medical University, 2-1-1-1 Midorigaoka-Higashi, Asahikawa, Hokkaido 078-8510, Japan; e-mail: h-mayumi@ asahikawamed.ac.jp

Junki Inamura, M.D., Ph.D.

Division of Gastroenterology and Hematology/Oncology, Department of Medicine, Asahikawa Medical University, 2-1-1-1 Midorigaoka-Higashi, Asahikawa, Hokkaido 078-8510, Japan; e-mail: junki@asahikawa-med.ac.jp Motohiro Shindo, M.D., Ph.D.

Division of Gastroenterology and Hematology/Oncology, Department of Medicine, Asahikawa Medical University, 2-1-1-1 Midorigaoka-Higashi, Asahikawa, Hokkaido 078-8510, Japan; e-mail: m-shindo@asahikawamed.ac.jp

Katsunori Sasaki, Ph.D.

Department of Gastrointestinal Immunology and Regenerative Medicine, Asahikawa Medical University, 2-1-1-1 Midorigaoka-Higashi, Asahikawa, Hokkaido 078-8510, Japan; e-mail: k-sasaki@ asahikawa-med.ac.jp

Naomi lizuka

Research and Development Department, Shino-Test Corporation, 2-29-14, Oonodai, Minamiku, Sagamihara, Kanagawa 229-0011, Japan; e-mail:

Naomi.Iizuka@shino-test.co.jp

Mikihiro Fujiya, M.D., Ph.D.

Division of Gastroenterology and Hematology/Oncology, Department of Medicine, Asahikawa Medical University, 2-1-1-1 Midorigaoka-Higashi, Asahikawa, Hokkaido 078-8510, Japan; e-mail: fjym@asahikawa-med.ac.jp Yoshihiro Torimoto, M.D., Ph.D. 
Oncology Center, Asahikawa Medical University Hospital, 2-1-1-1

Midorigaoka-Higashi, Asahikawa, Hokkaido 078-8510, Japan; e-mail:

tori@asahikawa-med.ac.jp

Yutaka Kohgo, M.D., Ph.D.

Division of Gastroenterology and Hematology/Oncology, Department of

Medicine, Asahikawa Medical University, 2-1-1-1 Midorigaoka-Higashi, Asahikawa, Hokkaido 078-8510, Japan; e-mail: yk1950@asahikawamed.ac.jp

\section{Authorship and Disclosures}

Contribution: S.I. and K.I. mainly and equally contributed to the study design, establishment of the quantification system, data analysis, and writing of the manuscript; D.K. and N.I. contributed to study design and establishment of the quantification system, and provided the reagents for the automated analyzer; H.T., L.A. and K. Sasaki contributed to NTBI quantification using non-metal HPLC; Y.T., M.H., J.I. and M.S. assisted with the study and obtained human serum samples; K. Shibusa and N.N. assisted with NTBI quantification using the automated analyzer; M.F., Y.T. and Y.K. contributed to data analysis and interpretation, and reviewed and provided comments on the manuscript; and all authors approved the final version of the manuscript.

Conflict-of-interest disclosure: S.I., K.I., Y.T., M.H., J.I., M.S., M.F., and Y.K. received research funding from Shino-Test Corporation for the collaborative research project with Shino-Test Corporation. Y.K. also received research funding from Novartis Pharma K.K. Other co-authors do not have a conflict of interest to disclose. 


\section{References}

[1] Andrews NC. Forging a field: the golden age of iron biology. Blood 2008;112(2):219-30.

[2] Kohgo Y, Ikuta K, Otake T, Torimoto T, Kato J. Body iron metabolism and pathophysiology of iron overload. Int J Hematol 2008;88(1):7-15.

[3] Garrick MD, Garrick LM. Cellular iron transport. Biochim Biophys Acta 2009;1790(5): 309-25.

[4] Hentze MW, Muckenthaler MU, Galy B, Camaschella C. Two to tango: Regulation of mammalian iron metabolism. Cell 2010;142(1):24-38.

[5] Hershko C, Graham G, Bates GW and Rachmilewitz EA: Non-specific serum iron in thalassaemia: an abnormal serum iron fraction of potential toxicity. $\mathrm{Br} \mathrm{J}$ Haematol 1978;40(2):255-63.

[6] Hider RC. Nature of nontransferrin-bound iron. Eur J Clin Invest 2002;32(Suppl.1):50-4.

[7] Xu X, Zhang L, Shen D, Wu H, Liu Q. Oxygen-dependent oxidation of Fe(II) to $\mathrm{Fe}(\mathrm{III})$ and interaction of $\mathrm{Fe}(\mathrm{III})$ with bovine serum albumin, leading to a hysteretic effect on the fluorescence of bovine serum albumin. J Fluoresc 2008;18(1):193-201.

[8] Grootveld M, Bell JD, Halliwell B, Aruoma OI, Bomford A, Sadler PJ. Nontransferrin-bound iron in plasma or serum from patients with idiopathic hemochromatosis. Characterization by high performance liquid chromatography and nuclear magnetic resonance spectroscopy. J Biol Chem 1989;264(8):4417-22.

[9] Esposito BP, Breuer W, Sirankapracha P, Pootrakul P, Hershko C, Cabantchik ZI. plasma iron in iron overload: redox activity and susceptibility to chelation. Blood 
2003;102(7):2670-7.

[10] Brissot P, Ropert M, Le Lan C, Loréal O. Non-transferrin bound iron: a key role in iron overload and iron toxicity. Biochim Biophys Acta 2012;1820(3):403-10.

[11] Cabantchik ZI(1), Breuer W, Zanninelli G, Cianciulli P. LPI-labile plasma iron in iron overload. Best Pract Res Clin Haematol. 2005;18(2):277-87.

[12] Hershko C: Mechanism of iron toxicity. Food Nutr Bull 2007;28(4 Suppl):S5009.

[13] Hershko C: Pathogenesis and management of iron toxicity in thalassemia. Ann N Y Acad Sci 2010;1202:1-9.

[14] Meo A, Ruggeri A, La Rosa MA, Zanghi L, Morabito N and Duca L: Iron burden and liver fibrosis decrease during a long-term phlebotomy program and iron chelating treatment after bone marrow transplantation. Hemoglobin 2006;30(1):131-7.

[15] Breuer W, Hershko C, Cabantchik ZI: The importance of non-transferrin bound iron in disorders of iron metabolism. Transfus Sci 2000;23(3):185-92.

[16] Gutteridge JM, Rowley DA, Halliwell B. Superoxide-dependent formation of hydroxyl radicals in the presence of iron salts. Detection of 'free' iron in biological systems by using bleomycin-dependent degradation of DNA. Biochem J 1981;199(1):263-5.

[17] Fargion S, Cappellini MD, Sampietro M, Fiorelli G. Non-specific iron in patients with beta-thalassaemia trait and chronic active hepatitis. Scand J Haematol $1981 ; 26(2): 161-7$.

[18] Singh S, Hider RC, Porter JB. A direct method for quantification of nontransferrin-bound iron. Anal Biochem 1990;186(2):320-3. 
[19] Mumby S, Koizumi M, Taniguchi N, Gutteridge JMC. Reactive iron species in biological fluids activate the iron-sulphur cluster of aconitase. Biochim Biophys Acta 1998;1380(1):102-8.

[20] Kolb AM, Smit NPM, Lentz-Ljuboje R, Osanto S, van Pelt J. Non-transferrin bound iron measurement is influenced by chelator concentration. Anal Biochem 2009;385(1):13-9.

[21] Gosriwatana I, Loreal O, Lu S, Brissot P, Porter J, Hider RC: Quantification of non-transferrin-bound iron in the presence of unsaturated transferrin. Anal Biochem 1999;273(2):212-20.

[22] Sasaki K, Ikuta K, Tanaka H, Ohtake T, Torimoto Y, Fujiya M, Kohgo Y. Improved quantification for non-transferrin-bound iron measurement using highperformance liquid chromatography by reducing iron contamination. Mol Med Rep 2011;4(5):913-8.

[23] Gutterridge KM, Quinlan GJ, Evans TW. Transient iron overload with bleomycin detectable iron in the plasma of patients with adult respiratory syndrome. Thorax 1994;49:707-10.

[24] Breuer W, Ermers MJ, Pootrakul P, Abramov A, Hershko C, Cabantchik ZI. Desferrioxamine-chelatable iron, a component of serum non-transferrin-bound iron, used for assessing chelation therapy. Blood 2001;97:792-98.

[25] Livrea MA, Tesoriere L, Pintaudi AM, Calabrese A, Maggio A, Freisleben HJ, et al. Oxidative stress and antioxidant status in beta-thalassemia major: iron overload and depletion of lipid-soluble antioxidants. Blood 1996;88:3608-14.

[26] Walter PB, Fung EB, Killilea DW, Jiang Q, Hudes M, Madden J, et al. Oxidative stress and inflammation in iron-overloaded patients with beta-thalassaemia or 
sickle cell disease. Br J Haematol 2006;135(2):254-63.

[27] Armbruster DA, Pry T. Limit of Blank, Limit of Detection and Limit of Quantitation. Clin Biochem Rev 2008;29(Suppl. 1):S49-52.

[28] Le Lan C, Loréal O, Cohen T, Ropert M, Glickstein H, Lainé F, et al. Redox active plasma iron in $\mathrm{C} 282 \mathrm{Y} / \mathrm{C} 282 \mathrm{Y}$ hemochromatosis. Blood 2005;105(11):4527-31.

[29] Breuer W, Cabantchik ZI. A fluorescence-based one-step assay for serum nontransferrin-bound iron. Anal Biochem 2001;299(2):194-202.

[30] Hunt JR, Zeng H. Iron absorption by heterozygous carriers of the HFE C282Y mutation associated with hemochromatosis. Am J Clin Nutr 2004;80(4):924-31.

[31] Dürken M, Nielsen P, Knobel S, Finckh B, Herrnring C, Dresow B, et al. Nontransferrin-bound iron in serum of patients receiving bone marrow transplants. Free Radic Biol Med 1997;22(7):1159-63.

[32] Lee DH, Liu DY, Jacobs DR Jr, Shin HR, Song K, Lee IK, et al. Common presence of non-transferrin-bound iron among patients with type 2 diabetes. Diabetes Care 2006;29(5):1090-5.

[33] von Bonsdorff L, Lindeberg E, Sahlstedt L, Lehto J, Parkkinen J. Bleomycindetectable Iron Assay for Non-Transferrin-bound Iron in Hematologic Malignancies. Clin Chem 2002;48:307-14.

[34] Loréal O, Gosriwatana I, Guyader D, Porter J, Brissot P, Hider RC. Determination of non-transferrin-bound iron in genetic hemochromatosis using a new HPLC-based method. J Hepatol 2000;32(5):727-33.

[35] Breuer W, Ronson A, Slotki IN, Abramov A, Hershko C, Cabantchik ZI. The assessment of serum non-transferrin-bound iron in chelation therapy and iron 
supplementation. Blood 2000;95(9):2975-82.

[36] Hilton RJ, Seare MC, Andros ND, Kenealey Z, Orozco CM, Webb M, Watt RK. Phosphate inhibits in vitro Fe3+ loading into transferrin by forming a soluble $\mathrm{Fe}(\mathrm{III})$-phosphate complex: a potential non-transferrin bound iron species. J Inorg Biochem 2012;110:1-7.

[37] Goto T, Ikuta K, Inamoto Y, Kamoshita S, Yokohata E, Koyama D, et al. Hyperferritinemia after adult allogeneic hematopoietic cell transplantation: quantification of iron burden by determining non-transferrin-bound iron. Int $\mathbf{J}$ Hematol. 2013;97(1):125-34. 


\section{Figure Legends}

Figure 1. Absorbance spectrum of iron-chelating chromogens complexed with metal ions. Black, red, green and blue lines correspond to $\mathrm{Fe}^{2+}, \mathrm{Cu}^{2+}$, $\mathrm{Co}^{2+}$ and $\mathrm{Ni}^{2+}$, respectively. (A) Nitroso-PSAP. (B)

Bathophenanthroline. (C) Ferene. (D) Ferrozine.

Figure 2. Reaction scheme of the automated NTBI assay system. Samples with NTBI are first mixed with Reagent- 1 to make a $[\mathrm{NTA}]_{2}-\mathrm{Fe}^{3+}$ complex. After incubation for $5 \mathrm{~min}$, Reagent- 2 is added to the reaction mixture. $[\mathrm{NTA}]_{2}-\mathrm{Fe}^{3+}$ is readily reduced and $\mathrm{Fe}^{2+}$ is released from the complex. Then nitroso-PSAP coordinates $\mathrm{Fe}^{2+}$ to form a nitroso-PSAP-Fe ${ }^{2+}$ complex. Abs750 of the resulting solution is measured.

Figure 3. NTBI levels in iron citrate, 5\% BSA and holo-Tf solutions were measured by the automated system. The total concentrations of iron in these solutions were $1.02 \mu \mathrm{M}, 1.04 \mu \mathrm{M}$ and $15 \mu \mathrm{M}$, respectively. The results are shown as the percentage of NTBI relative to the total iron concentrations. Values are shown as means \pm S.D. $(n=5)$.

Figure 4. Linearity was assessed up to $20 \mu \mathrm{M}$ NTBI. The NTBI level measured by the automated system was plotted against the concentration of iron citrate. Values are shown as means \pm S.D. $(n=5)$. 
Figure 5. (A) The relationship between transferrin saturation (open circle) and the NTBI level (closed square) was determined by adding standard iron solutions $(0-75 \mu \mathrm{M})$ to pooled human serum. (B) Comparison of transferrin saturation and NTBI levels in 25 patients.

Figure 6. Interference by hemolysis was examined with the assay system. Values are shown as means \pm S.D. $(n=5)$.

Figure 7. NTBI levels in sera from 33 patients (A) and NTA-Fe (B) were quantified by HPLC and the automated NTBI assay system. Data from (A) and (B) are combined and shown as (C). 

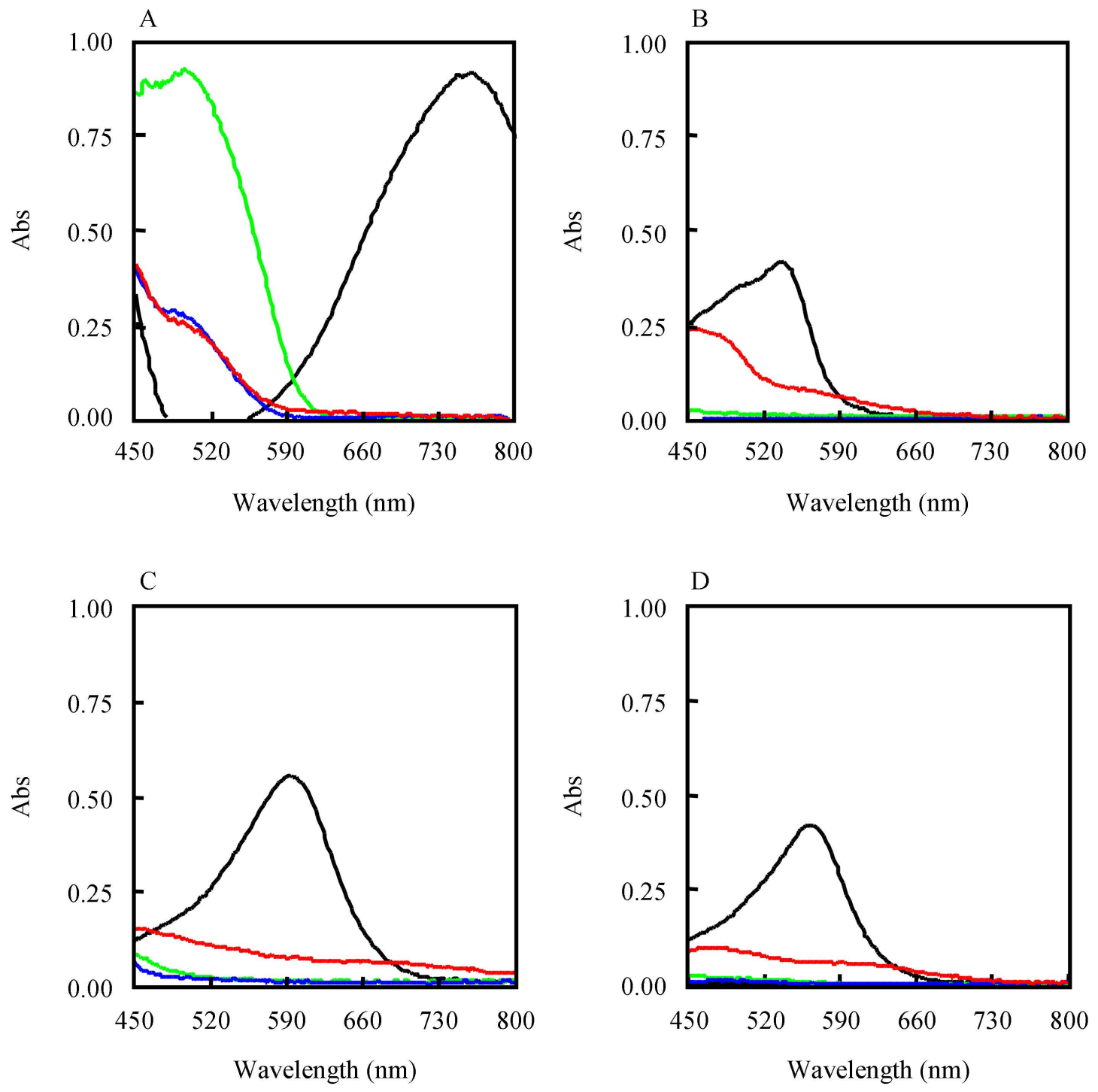

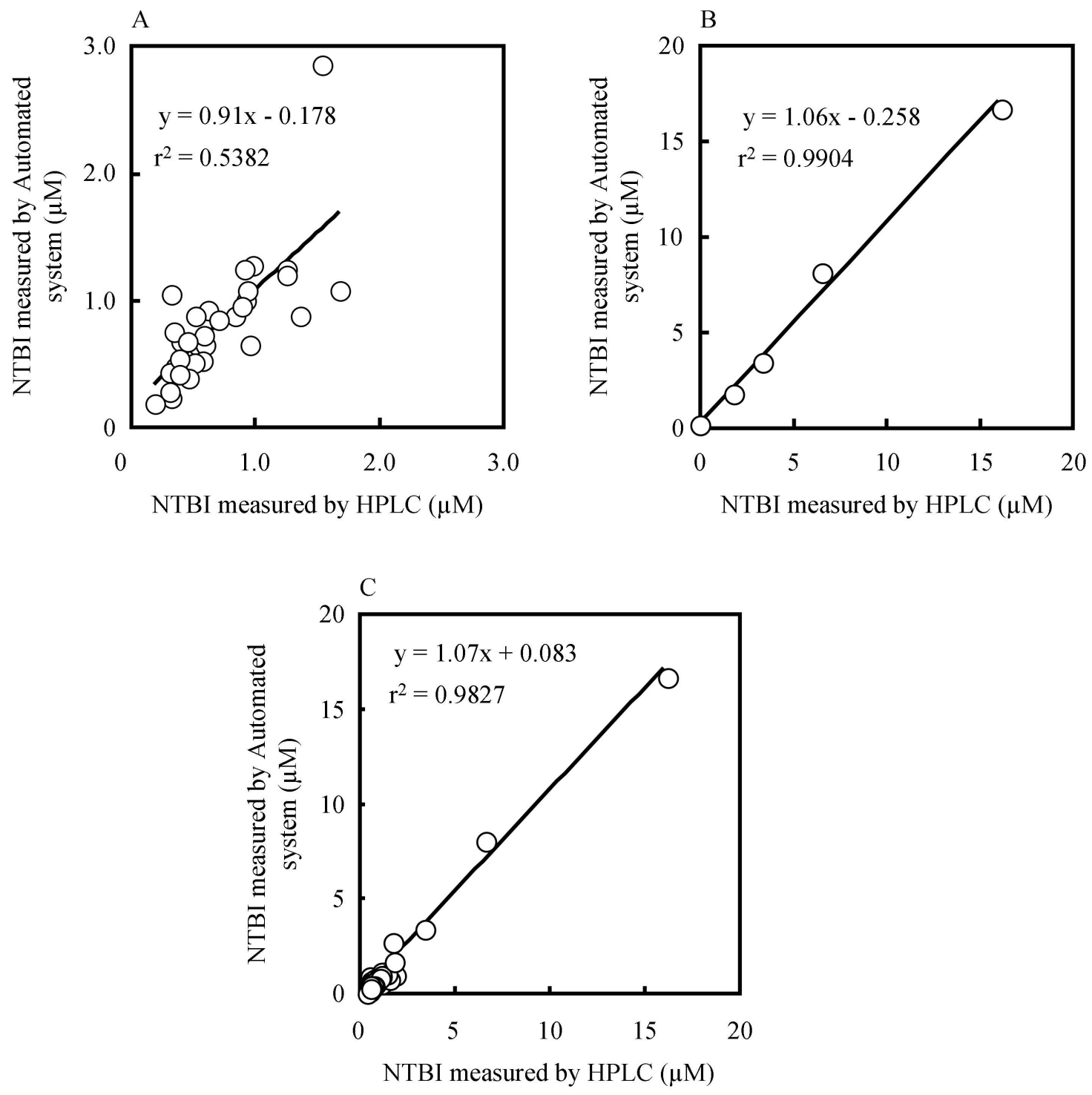


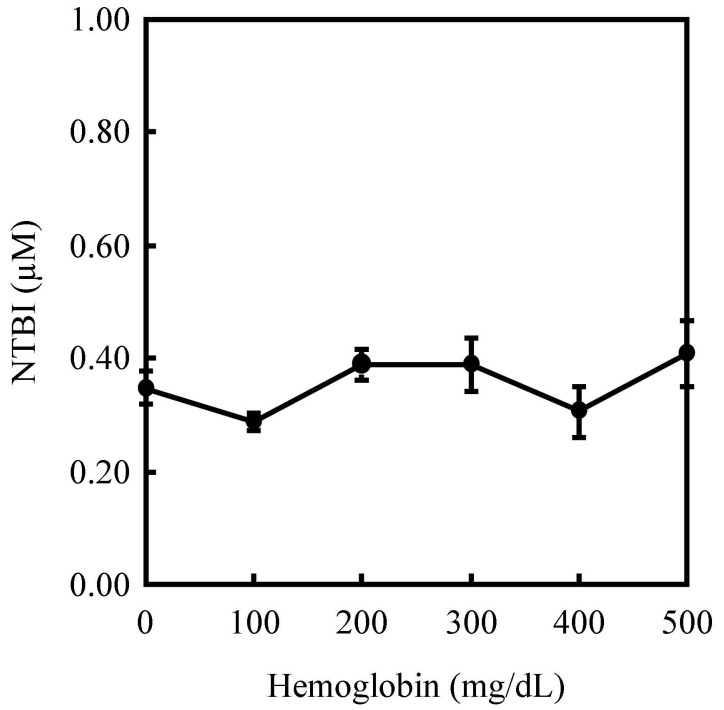



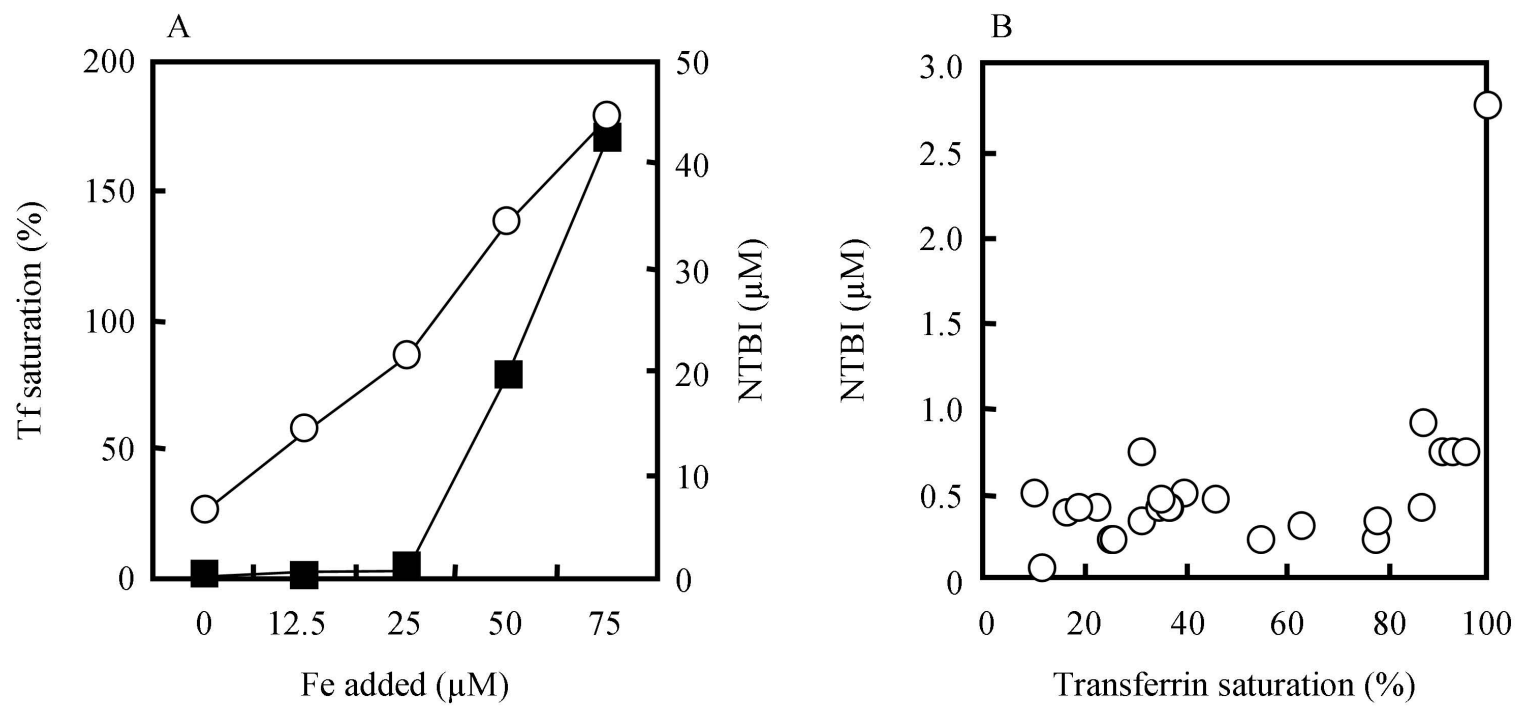


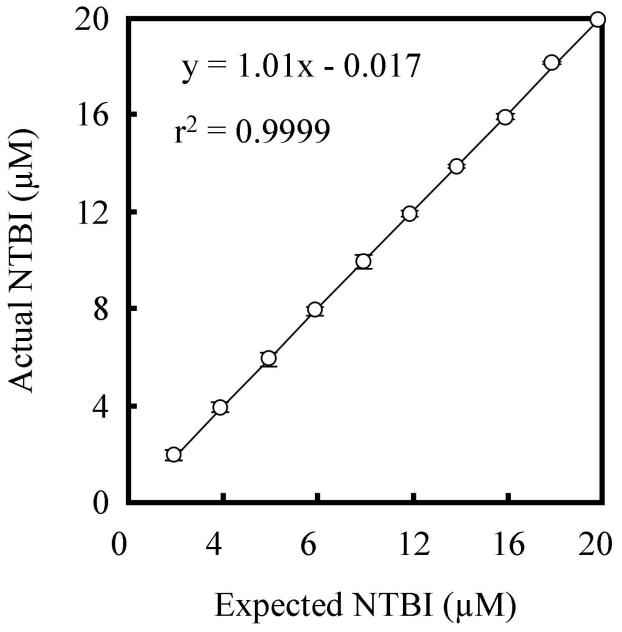




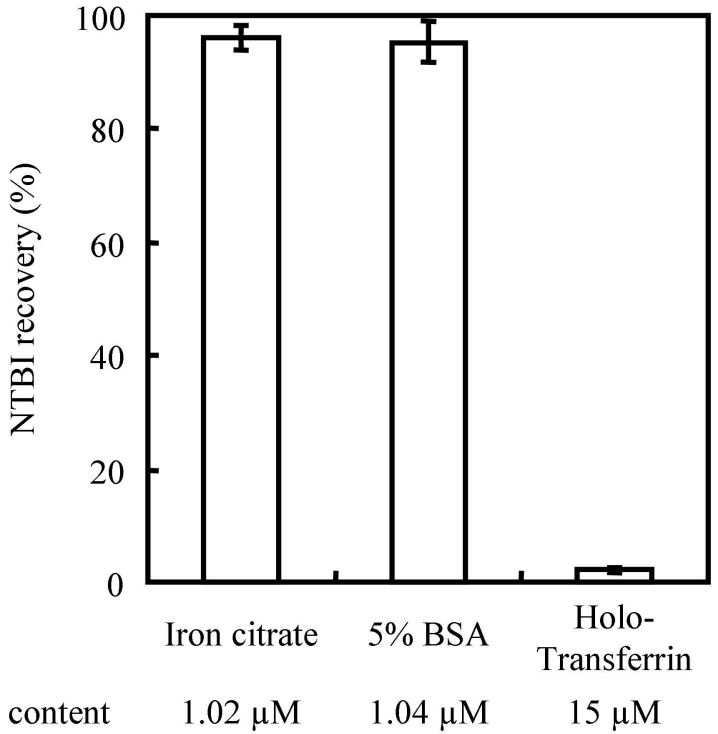




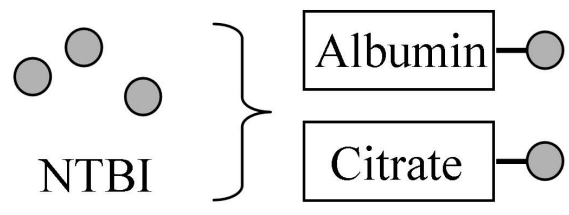

$$
+\mathrm{NTA} \square[\mathrm{NTA}]_{2}-\mathrm{Fe}^{3+}
$$

$<$ Sample>

Reagent-2 (Nitroso-

Ascorbic acid PSAP)

Measure $\mathrm{Abs}_{750}$

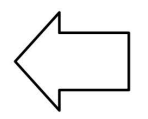

Nitroso-PSAP $-\mathrm{Fe}^{2+}$

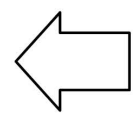

$\mathrm{Fe}^{2+}$

Nitroso-PSAP 